\title{
Stretchable Persistent Spin Helices in GaAs Quantum Wells
}

\author{
Florian Dettwiler, ${ }^{1}$ Jiyong Fu, ${ }^{2, \dagger}$ Shawn Mack, ${ }^{3, \$}$ Pirmin J. Weigele, ${ }^{1}$ J. Carlos Egues, ${ }^{2}$ \\ David D. Awschalom, ${ }^{3,4}$ and Dominik M. Zumbühl ${ }^{1, *}$ \\ ${ }^{1}$ Department of Physics, University of Basel, CH-4056 Basel, Switzerland \\ ${ }^{2}$ Instituto de Física de São Carlos, Universidade de São Paulo, 13560-970 São Carlos, São Paulo, Brazil \\ ${ }^{3}$ California NanoSystems Institute, University of California, Santa Barbara, California 93106, USA \\ ${ }^{4}$ Institute for Molecular Engineering, University of Chicago, Chicago, Illinois 60637, USA \\ (Received 16 February 2017; revised manuscript received 21 May 2017; published 18 July 2017)
}

The Rashba and Dresselhaus spin-orbit (SO) interactions in 2D electron gases act as effective magnetic fields with momentum-dependent directions, which cause spin decay as the spins undergo arbitrary precessions about these randomly oriented SO fields due to momentum scattering. Theoretically and experimentally, it has been established that by fine-tuning the Rashba $\alpha$ and renormalized Dresselhaus $\beta$ couplings to equal fixed strengths $\alpha=\beta$, the total SO field becomes unidirectional, thus rendering the electron spins immune to decay due to momentum scattering. A robust persistent spin helix (PSH), i.e., a helical spin-density wave excitation with constant pitch $P=2 \pi / Q, Q=4 m \alpha / \hbar^{2}$, has already been experimentally realized at this singular point $\alpha=\beta$, enhancing the spin lifetime by up to 2 orders of magnitude. Here, we employ the suppression of weak antilocalization as a sensitive detector for matched SO fields together with independent electrical control over the SO couplings via top gate voltage $V_{T}$ and back gate voltage $V_{B}$ to extract all SO couplings when combined with detailed numerical simulations. We demonstrate for the first time the gate control of the renormalized $\beta$ and the continuous locking of the SO fields at $\alpha=\beta$; i.e., we are able to vary both $\alpha$ and $\beta$ controllably and continuously with $V_{T}$ and $V_{B}$, while keeping them locked at equal strengths. This makes possible a new concept: "stretchable PSHs," i.e., helical spin patterns with continuously variable pitches $P$ over a wide parameter range. Stretching the PSH, i.e., gate controlling $P$ while staying locked in the PSH regime, provides protection from spin decay at the symmetry point $\alpha=\beta$, thus offering an important advantage over other methods. This protection is limited mainly by the cubic Dresselhaus term, which breaks the unidirectionality of the total SO field and causes spin decay at higher electron densities. We quantify the cubic term, and find it to be sufficiently weak so that the extracted spin-diffusion lengths and decay times show a significant enhancement near $\alpha=\beta$. Since within the continuous-locking regime quantum transport is diffusive (2D) for charge while ballistic (1D) for spin and thus amenable to coherent spin control, stretchable PSHs could provide the platform for the much heralded long-distance communication $\sim 8-25 \mu$ m between solid-state spin qubits, where the spin diffusion length for $\alpha \neq \beta$ is an order of magnitude smaller.

DOI: 10.1103/PhysRevX.7.031010

The inextricable coupling between the electron spatial and spin degrees of freedom - the spin-orbit (SO) interactionunderlies many fundamental phenomena such as the spin Hall effects—quantum and anomalous [1] — and plays a crucial role in newly discovered quantum materials hosting

\footnotetext{
*dominik.zumbuhl@unibas.ch

Permanent address: Department of Physics, Qufu Normal University, Qufu, Shandong, 273165, China.

${ }^{\ddagger}$ Present address: Naval Research Laboratory, Washington, D.C. 20375 , USA.

Published by the American Physical Society under the terms of the Creative Commons Attribution 4.0 International license. Further distribution of this work must maintain attribution to the author(s) and the published article's title, journal citation, and DOI.
}

Subject Areas: Condensed Matter Physics, Quantum Information, Spintronics

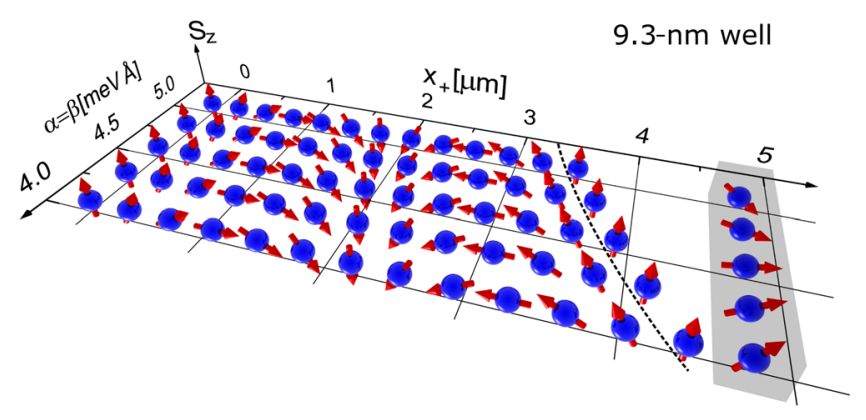

FIG. 1. Stretchable PSHs. Illustration of spin helices at different values of $\alpha=\beta$ accessible in the measurements. The position $x_{+}$for one $2 \pi$ rotation (dashed curve) is changing for the gate-locked regime $\alpha=\beta$. The gray box highlights how the spin rotation can be controlled (in situ) at fixed position $\sim 4.8 \mu \mathrm{m}$ by $\sim \pi / 2$ over the same range of $\alpha=\beta$. The $\hat{x}_{+} \|[110]$ and $\hat{x}_{-} \|[\overline{1} 10]$ axes define the $2 \mathrm{D}$ plane. 
Majorana [2] and Weyl fermions [3]. In nanostructures, the SO coupling strength can be varied via gate electrodes $[4,5]$. As recently demonstrated [6], this enables controlled spin modulation [7] of charge currents in nonmagnetic (quasiballistic) spin transistors.

The SO coupling in a GaAs quantum well has two dominant contributions: the Rashba [8] and the Dresselhaus [9] effects, arising from the breaking of the structural and crystal inversion symmetries, respectively. When the Rashba $\alpha$ and Dresselhaus $\beta$ SO couplings match at $\alpha=\beta[10,11]$, the direction of the combined RashbaDresselhaus field becomes momentum independent, thus suppressing D'yakonov-Perel spin-flip processes, provided that the cubic Dresselhaus term be small. The significantly enhanced spin lifetime at $\alpha=\beta$ enables nonballistic spin transistors and persistent spin helices (PSHs) [10,11]. However, despite substantial efforts, so far this symmetry point has been achieved only at isolated points with finely tuned system parameters [12-14], which is too difficult to be reliably attained on demand as required for a useful technology.

Stretchable persistent spin helices.-Here, we overcome this outstanding obstacle by (i) using a technique that allows independent control of the SO couplings via a top gate voltage $V_{T}$ and a back gate voltage $V_{B}$ while (ii) simultaneously measuring the suppression of weak antilocalization (WAL) in an external magnetic field as a sensitive probe for matched SO couplings. While gate tuning of the renormalized Dresselhaus coefficient $\beta$ was already theoretically described in 1994 [15], we demonstrate this for the first time here in an experiment, and employ this tunability to show robust continuous locking of the Rashba and Dresselhaus couplings at $\alpha\left(V_{T}, V_{B}\right)=$ $\beta\left(V_{T}, V_{B}\right)$ over a wide range of densities $n$, i.e., a "symmetry line" (not a point) in the $\left(V_{T}, V_{B}\right)$ plane. This allows us to introduce the concept of the "stretchable persistent spin helix," see Fig. 1, with spin density $s_{x_{+}} \sim \sin \left(Q x_{+}\right), s_{x_{-}}=0$, and $s_{z} \sim \cos \left(Q x_{+}\right)$and variable pitch $P=2 \pi / Q, Q=4 m \alpha / \hbar^{2}$. The stretchable PSH makes possible gate control of the spin precession over long distances due to strong protection from spin decay by up to 2 orders of magnitude enhanced spin lifetimes at the symmetry point $\alpha=\beta$-without requiring in-plane electric fields to induce drift [16], and without relying on micron-width channels to suppress decay [17].

Long-distance spin communication.-Within the range of the continuously matched-locked SO couplings $\alpha=\beta$, quantum transport in the well is diffusive for charge (2D) while essentially ballistic (1D) for spins [see Supplemental Material (SM), Sec. V [18]]. A stretchable PSH could thus be used to coherently couple, e.g., spin qubits over unprecedented long distances. Figure 1 illustrates how spin information can be conveyed between spins via a stretchable PSH. These stretchy waves can be excited upon injection of spin polarization; see, e.g., Refs. [12,13]. Other spin communication modes can be envisaged with this setup. The distance is limited mainly by the deviation from $\alpha=\beta$ and by the cubic Dresselhaus term, which is small in this range, as we quantify later on, and leads to spin decay with spin-diffusion lengths $\lambda_{\text {eff }} \sim 8-25 \mu \mathrm{m}$ over which the spin dephases by $1 \mathrm{rad}$. Note that this type of spin manipulation and spin transfer is not possible for a helix with $\alpha \neq \beta$, since $\lambda_{\text {eff }}$ quickly drops below the helix pitch as the SO couplings are deviating from the symmetry point.

The full electrical control of the SO couplings demonstrated in our 9.3-nm-wide quantum well can tune from $\alpha=\beta=5 \mathrm{meV} \AA$ to $4 \mathrm{meV} \AA$, thus enabling stretchable PSHs with pitches $P$ stretching from 3.5 to $4.4 \mu \mathrm{m}$; see Fig. 1. Within the shortest spin-diffusion length $\lambda_{\text {eff }} \sim 8 \mu \mathrm{m}$ for our 9.3-nm well, controlled spin rotations by an angle $\theta=Q x_{+}=2 \pi x_{+} / P$ can be performed under spin protection on any spin sitting at a position $x$ along the stretchable PSH by varying $P$ in the range above. For example, a spin at $x \sim 4.8 \mu \mathrm{m}$ can be rotated by $\Delta \theta \sim \pi / 2$ as $P$ varies in the range above; see gray box shading in Fig. 1. Thus, stretchable helices could provide a platform for longdistance spin communication.

Additional results.-WAL is also used to identify other regimes such as the Dresselhaus regime $\alpha=0$ in a more symmetrically doped sample. Combined with numerical simulations, we extract the SO couplings $\alpha$ and $\beta$, the bulk Dresselhaus parameter $\gamma$, the spin-diffusion lengths, and spin-relaxation times over a wide range of system parameters. We also quantify the detrimental effects of the third harmonic of the cubic Dresselhaus term, which mainly limits spin protection. Interestingly, our spindiffusion lengths and spin-relaxation times are significantly enhanced within the locked $\alpha=\beta$ range, thus attesting that our proposed setup offers a promising route for spin protection and manipulation.

In what follows, we first explain tuning of the Rashba coupling, then the essential density dependence of the Dresselhaus coupling $\beta$ that enables the continuous locking of the SO fields, how it also leads to spin decay at higher densities, followed by the relevant weak-localizationweak-antilocalization (WL-WAL) detection scheme, measurements, and simulations. A full account of our approach, including additional data and details of the model and simulations, is presented in the Appendix and the SM [18].

Controlling the Rashba coupling $\alpha$.- The Rashba coefficient [8] $\alpha$ can be tuned with the wafer and doping profile [12] as well as in situ using gate voltages [4,5] at constant density $n$ and thus independent of the Dresselhaus term; see below. A change of top gate voltage $V_{T}$ can be compensated by an appropriate, opposing change of back gate voltage $V_{B}$ [see Fig. 2(a)] to keep $n$ fixed $[19,20]$ while changing the gate-induced electric field $\delta E_{Z}$ in the quantum well, where $z \perp 2 \mathrm{D}$ plane. Another Rashba term due to 
9.3-nm QW, asymmetrically doped
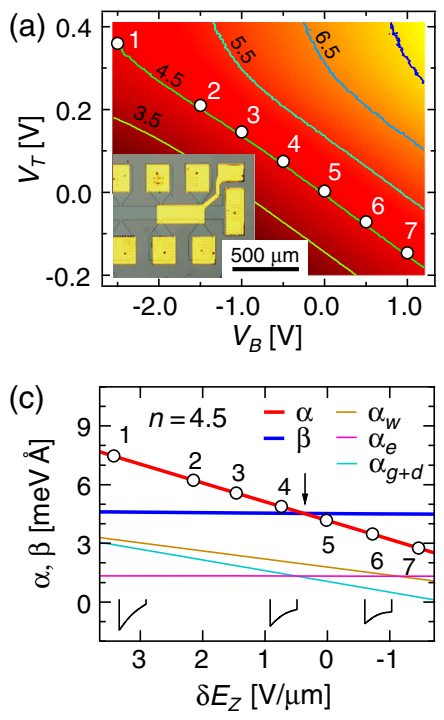

FIG. 2. Weak localization (WL) as an $\alpha=\beta$ detector, gate control of Rashba $\alpha$ at constant density. (a) Measured charge density $n$ (color) versus top gate voltage $V_{T}$ and back gate voltage $V_{B}$ (9.3-nm well). Contours of constant density (3.5-7.5) $\times$ $10^{11} \mathrm{~cm}^{-2}$ are shown. Inset: Optical micrograph of typical Hall bar, with contacts (yellow), gate (center), and mesa (black lines). (b) Normalized longitudinal conductivity $\Delta \sigma / \sigma_{0}=\left[\sigma\left(B_{Z}\right)-\right.$ $\sigma(0)] / \sigma(0)$ versus $B_{Z} \perp 2 \mathrm{D}$ plane. Curves for gate configurations 1-7 along constant $n=4.5 \times 10^{11} \mathrm{~cm}^{-2}$ are shown (offset vertically), also labeled in (a) and (c). (c) Simulated Rashba $\alpha$ and Dresselhaus $\beta$ coefficients (see text) against gate-induced field change $\delta E_{Z}$, shown for constant $n=4.5 \times 10^{11} \mathrm{~cm}^{-2}$. The $\delta E_{Z}$ axis-decreasing from left to right—corresponds exactly to the $V_{B}$ abscissa of (a) for a covarying $V_{T}$, such that $n=4.5 \times$ $10^{11} \mathrm{~cm}^{-2}$ constant. Sketches of the well potential at 1,4 , and 6 illustrate the change of $\alpha$ with $\delta E_{Z}$. Note that $\alpha\left(\delta E_{Z}=0\right) \neq 0$ since the external $E$ field (see SM [18]) is not zero at $\delta E_{Z}=0$.

donor electric fields [21,22] is negligible in our structures; see SM [18].

Linear and cubic Dresselhaus terms in 2D.-Because of the well confinement along the $z$ direction (growth), the cubic-in-momentum bulk (3D) Dresselhaus SO interaction gives rise to, after the projection into the lowest quantum well subband eigenstates, distinct terms that are linear and cubic in $\mathbf{k}$, the $2 \mathrm{D}$ electron wave vector. The linear-in- $k$ term has a coefficient $\beta_{1}=\gamma\left\langle k_{z}^{2}\right\rangle$ and turns out practically independent of density in the parameter range of interest here. The cubic-in- $k$ term, on the other hand, is density dependent and has yet two components with distinct angular symmetries: (i) the first-harmonic contribution proportional to $\sin \phi$ and $\cos \phi$ and (ii) the third-harmonic contribution proportional to $\sin 3 \phi$ and $\cos 3 \phi$; here, $\phi$ is the polar angle in $2 \mathrm{D}$ between $\mathbf{k}$ and the [100] direction (see SM [18]). Interestingly, the first-harmonic contribution with coefficient $\beta_{3}$ has the same angular symmetry as both the linear-in- $k$ Dresselhaus $\beta_{1}$ term (see Refs. $[15,23]$ ) and

the Rashba $\alpha$ term. An additional term with the same form-the interface Dresselhaus term [24]—could also play a role; see SM [18].

To a very good approximation, the coefficient $\beta_{3} \simeq$ $\gamma k_{F}^{2} / 4$, where the Fermi vector $k_{F} \simeq \sqrt{2 \pi n}$ and $n$ is the carrier density of the 2D gas. This neglects the tiny angular anisotropy in the Fermi wave vector due to the competition between the Rashba and Dresselhaus effects (especially in GaAs wells). Note that by approximating $\beta_{3} \simeq \gamma \pi n / 2$, both the first-harmonic and the third-harmonic parts of the cubic-in- $k$ Dresselhaus term actually become linear in $k$ (see SM for details [18]) and, more importantly, become density dependent. We can now group the linear-in- $k$ Dresselhaus term $\beta_{1}$ together with the first-harmonic contribution $\beta_{3}$ into a single renormalized Dresselhaus term by defining $\beta=\beta_{1}-\beta_{3}$. It is this density-dependent renormalized coefficient $\beta$ that can be tuned with a gate voltage to match the Rashba $\alpha$ coupling continuously. This matching leads to a $k$-independent spinor (or, equivalently, to a $k$-independent effective SO field), whose direction is immune to momentum scattering. In this way we achieve independent, continuous control of the Rashba and Dresselhaus terms by using top gate and back gate voltages. This is an unprecedented tunability of the SO terms within a single sample.

Spin decay at higher densities. - The strength of the third-harmonic contribution of the Dresselhaus term is also described by the coefficient $\beta_{3}$. This term, however, is detrimental to spin protection as it breaks the angular symmetry of the other linear SO terms and makes the spinor $k$ dependent and susceptible to in-plane momentum scattering, even for matched couplings $\alpha=\beta$. As we show, the detrimental effect of the third-harmonic contribution does not prevent our attaining the continuous locking over a relevant wide range of electron densities.

Gate-tunable range of the Dresselhaus coupling $\beta$.-For the narrow quantum wells we use here, $\beta_{1}$ is essentially gate independent since the wave function spreads over the full width of the well. This also implies $\left\langle k_{z}^{2}\right\rangle \ll(\pi / W)^{2}$ (the infinite well limit), see Fig. 3(d), due to wave function penetration into the finite barriers. Thus, a change of density by a factor of $\sim 2.5$ changes $\beta_{3} / \beta_{1}=\pi n /\left\langle 2 k_{z}^{2}\right\rangle$ by the same factor, resulting in a gate-tunable range of $0.08 \lesssim \beta_{3} / \beta_{1} \lesssim 0.2$. In addition, quantum wells of width $W=8,9.3,11$, and $13 \mathrm{~nm}$ were used [12,25], resulting in a change of $\beta_{1}$ by roughly a factor of 2 .

Detection scheme for matched $S O$ couplings.-WAL is a well-established signature of SO coupling in magnetoconductance $\sigma\left(B_{Z}\right)[15,23,26-29]$ exhibiting a local maximum at zero field. In the $\alpha= \pm \beta$ regime, the resulting internal SO field is uniaxial, and spin rotations commute and are undone along time-reversal loops. Therefore, WAL is suppressed and the effectively spinless situation displaying weak localization [i.e., $\sigma\left(B_{Z}\right)$ exhibiting a local minimum at $\left.B_{Z}=0\right]$ is restored [10,11,14,23]. Away from the 

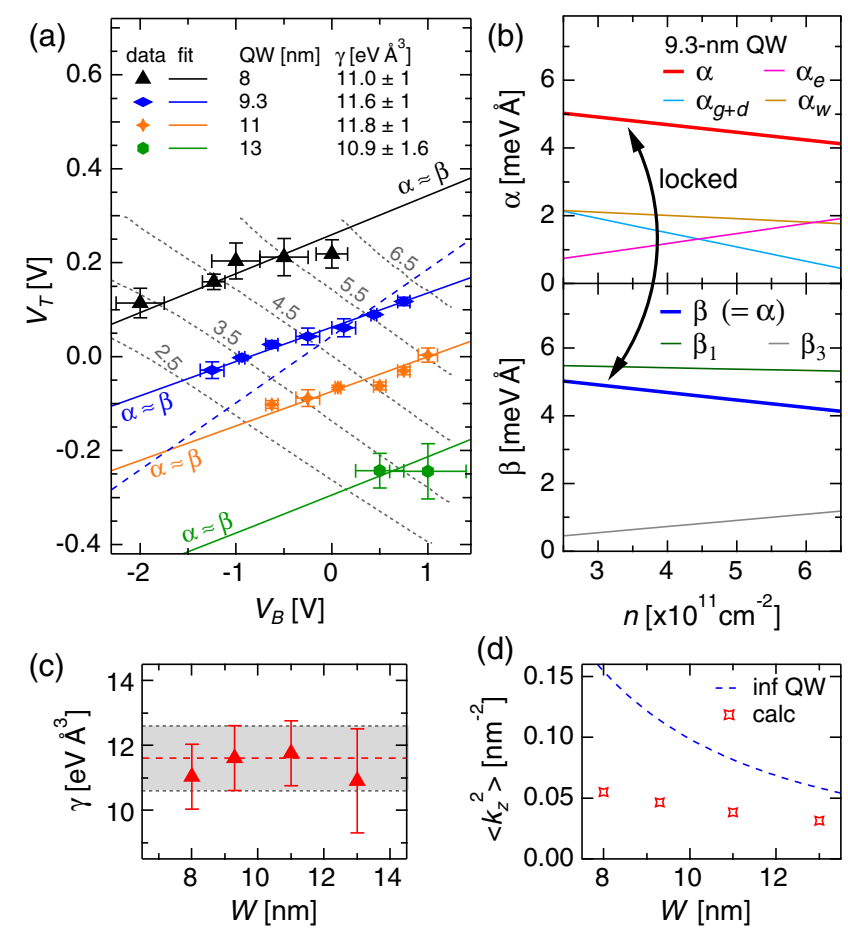

(d)

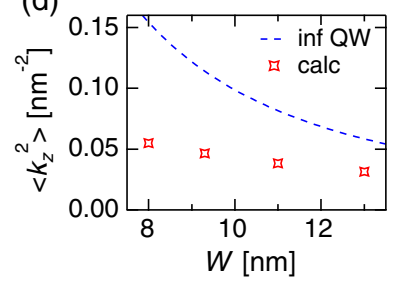

FIG. 3. Tuning and continuously locking $\alpha=\beta$. (a) The markers indicate $\alpha \approx \beta$ for four different well widths (asymmetric doping) and various densities (gray contours of constant $n$, labeled in units of $10^{11} \mathrm{~cm}^{-2}$ ) in the $V_{T}$ and $V_{B}$ plane. Error bars result from the finite number of conductance traces in the $\left(V_{B}, V_{T}\right)$ space. Theory fits (solid lines) are shown for each well, with $\gamma$ as the only fit parameter (inset table, error bars dominated by systematic error; see below). The dashed blue line indicates the slope of constant $\alpha=\beta_{1}$, neglecting $\beta_{3}$, which is inconsistent with the data. (b) Simulation of locked $\alpha=\beta$ versus density $n$ along solid blue line from (a), showing the various SO contributions (see text). (c) Values of $\gamma$ from fits for each well width $W$. Red dashed line is the average $\gamma=11.6 \pm 1 \mathrm{eV} \AA^{3}$ (excluding $W=13 \mathrm{~nm}$ due to its larger error), gray area is the $\sim 9 \%$ error, stemming mostly from the systematic uncertainty in the input parameters of the simulations (see Appendix). (d) $\left\langle k_{z}^{2}\right\rangle$ as a function of well width $W$ for realistic (markers) and infinite (blue) potential.

matched regime, the SO field is not uniaxial, spin rotations do not commute, and trajectories in time-reversal loops interfere destructively upon averaging [26] due to the SO phases picked up along the loops, thus leading to WAL. Hence, this suppression is a sensitive detector for $\alpha= \pm \beta$. At high $\beta_{3}$, this detection scheme becomes approximate, giving $\alpha= \pm \beta$ [14]. We note that the WL dip—often used to determine phase coherence-sensitively depends on the SO coupling [e.g., curves 3-6 in Fig. 2(b)], even before WAL appears. Negligence of SO coupling could thus lead to spurious or saturating coherence times. At higher temperatures, when quantum coherence is lost, this detection scheme becomes inoperable, while it is expected that the mechanism for tuning both Rashba and effective Dresselhaus coefficients continues to function with only small corrections $[24,30]$ even up to room temperature. Also, note that Shubnikov-de Haas oscillations do not show any spin-orbit splitting here (see SM [18]) given the strength of SO coupling in GaAs, making it clear that the quantum corrections in WAL and their suppression at the symmetry point present a very sensitive detector for SO coupling.

Continuous locking $\alpha=\beta$.-We proceed to demonstrate gate locking of the $\mathrm{SO}$ couplings $\alpha, \beta$. Figure 2(b) displays $\sigma\left(B_{Z}\right)$ of the 9.3-nm well for top gate and back gate configurations labeled $1-7$, all lying on a contour of constant density; see Fig. 2(a). Along this contour, $\beta$ is held fixed since the density is constant $\left(\beta_{1}\right.$ is essentially gate independent), while $\alpha$ is changing as the gate voltages are modifying the electric field $\delta E_{Z}$ perpendicular to the quantum well. Across these gate configurations, the conductance shows a transition from WAL (configuration 1 and 2) to WL (4 and 5) back to WAL (7). Selecting the most pronounced WL curve allows us to determine the symmetry point $\alpha=\beta$. This scheme is repeated for a number of densities, varying $n$ by a factor of 2 , yielding the symmetry point $\alpha=\beta$ for each density $n$ [see Fig. 3(a), blue markers], thus defining a symmetry line in the $\left(V_{T}, V_{B}\right)$ plane. Along this line, $\beta$ is changing with density, as previously described, and $\alpha$ follows $\beta$, remaining "continuously" locked at $\alpha=\beta$. As mentioned earlier, this is a very interesting finding, as it should allow the creation of persistent spin helices with gate-controllable pitches, as illustrated in Fig. 1.

Simulations and fitting of $\gamma$.- - Self-consistent calculations combined with the transport data can deliver all SO parameters. The numerical simulations [31] (see Appendix and SM [18]) can accurately calculate $\alpha$ and $\left\langle k_{z}^{2}\right\rangle$. This leaves only one fit parameter, $\gamma$, the bulk Dresselhaus coefficient, which can now be extracted from fits to the density dependence of the symmetry point, see solid blue line in Fig. 3(a), giving excellent agreement with the data (blue markers). This procedure can be repeated for a set of wafers with varying quantum well width and thus varying $\beta_{1}$. This shifts the symmetry point $\alpha=\beta$, producing nearly parallel lines, as indicated with colors in Fig. 3(a) corresponding to the various wafers as labeled. As shown, locking $\alpha=\beta$ over a broad range is achieved in all wafers. Since gate voltages can be tuned continuously, any and all points on the symmetry lines $\alpha=\beta$ can be reached. Again performing fits over the density dependence of the symmetry point for each well width, we obtain very good agreement, see Fig. 3(a), and extract $\gamma=11.6 \pm 1 \mathrm{eV} \AA^{3}$ consistently for all wells [Fig. 3(c)]. We emphasize that $\gamma$ is notoriously difficult to calculate and measure [28,29,32]; the value we report here agrees well with recent studies $[13,32,33]$. Obtaining consistent values over wide ranges of densities and several wafers with varying well widths provides a robust method to extract $\gamma$.

Beyond $\gamma$, the simulations reveal important information about the gate tuning of the SO parameters. The Rashba 
coefficient is modeled as $\alpha=\alpha_{g+d}+\alpha_{w}+\alpha_{e}$ in the simulation, with gate and doping term $\alpha_{g+d}$, quantum well structure term $\alpha_{w}$, and Hartree term $\alpha_{e}$. Along a contour of constant density, the simulations show that mainly $\alpha_{g+d}$ and $\alpha_{w}$ are modified, while $\alpha_{e}$ and $\beta$ remain constant; see Fig. 2(c). The density dependence for locked $\alpha=\beta$, on the other hand, shows that while $\beta_{1}$ is nearly constant, $\beta_{3}$ is linearly increasing with $n$, thus reducing $\beta=\beta_{1}-\beta_{3}$; see Fig. 3(b). Hence, to keep $\alpha=\beta$ locked, $\alpha$ has to be reduced correspondingly. The Hartree term $\alpha_{e}$, however, increases for growing $n$. Thus, on the $\alpha=\beta$ line, the other $\alpha$ termsmainly the gate-dependent $\alpha_{g+d}$-are strongly reduced, maintaining locked $\alpha=\beta$, as shown in Fig. 3(b). We emphasize that neglecting the density dependence of $\beta_{3}$ and fixing $\alpha=\beta_{1}+$ const results in a line with slope indicated by the blue dashed line in Fig. 3(a), which is clearly inconsistent with the data. Thus, the densitydependent $\beta_{3}$ enabling gate tunability of the Dresselhaus term is crucial here.

Dresselhaus regime.-We now show that $\alpha$ can be tuned through $\beta$ and through zero in a more symmetrically doped wafer, opening the Dresselhaus regime $\beta \gg \alpha$. We introduce the magnetic field $B_{\mathrm{SO}}$, where the magnetoconductance exhibits minima at $B_{Z 1} \approx-B_{Z 2}$. These minima describe the crossover between WAL and WL, where the Aharonov-Bohm dephasing length and the SO diffusion length are comparable. Beyond the WAL-WL-WAL transition [Fig. 4(b), upper panel], $B_{\text {SO }}$ is seen to peak and decrease again (dashed curve). The gate voltages with maximal $B_{\text {SO }}$ are added to Fig. 4(a) for several densities (red markers). We surmise that these points mark $\alpha \approx 0$ : $B_{\text {SO }}$ signifies the crossover from WL to WAL-like conductance, thus defining an empirical measure for the effects of SO coupling (larger $B_{\mathrm{SO}}$, stronger effects). For $\alpha=0$, the full effect of $\beta$ on the conductance becomes apparent without cancellation from $\alpha$, giving a maximal $B_{\mathrm{SO}}$. Indeed, the simulated $\alpha=0$ curve [dashed red line in Fig. 4(a)] cuts through the experimental points, also reflected in Fig. 4(c) by a good match with the simulated $\alpha=0$ crossing point (red arrow).

Diverging spin-orbit lengths.-For a comparison of experiment and simulation, we convert the empirical $B_{\mathrm{SO}}$ to a "magnetic length" $\lambda_{\mathrm{SO}}=\sqrt{\hbar / 2 e B_{\mathrm{SO}}}$, which we later on interpret as a spin-diffusion length, where $e>0$ is the electron charge and the factor of 2 accounts for timereversed pairs of closed trajectories. We also introduce the ballistic SO lengths $\lambda_{ \pm}=\hbar^{2} /\left(2 m^{*}|\alpha \pm \beta|\right)$. These lengths correspond to a spin rotation of $1 \mathrm{rad}$, as the electrons travel along $\hat{x}_{+}$and $\hat{x}_{-}$, respectively, with spins initially aligned perpendicular to the corresponding SO field [e.g., for an electron moving along the $\hat{x}_{+}$, its spin should point along $\hat{x}_{+}$or $\hat{z}$ so spin precession can occur; see SM, Eq. (S20), for an expression of the SO field [18]]. For $\alpha=+\beta$, $\lambda_{-}$diverges (no precession, indicating that an electron traveling along $\hat{x}_{-}$does not precess) while $\lambda_{+}$is finite, and
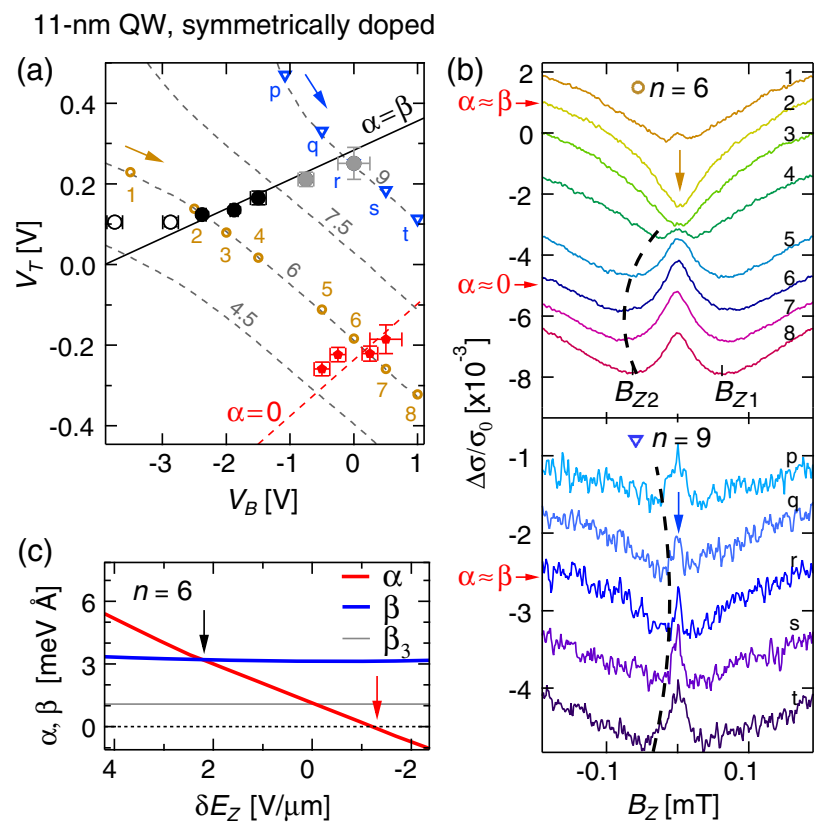

FIG. 4. The Dresselhaus and the cubic regime. (a) Locked regime $\alpha \approx \beta$ (black or gray symbols) and Dresselhaus regime $\alpha \approx 0$ (red symbols) from the broadest WAL minima (maximal $B_{\mathrm{SO}}$ ) in the $V_{T}$ and $V_{B}$ plane for a more symmetrically doped 11-nm well. The solid black line displays the $\alpha=\beta$ simulation, while the dashed red line marks the simulated $\alpha=0$ contour. Open black markers (leftmost $V_{B}$ ) are entering the nonlinear gate regime, causing a slight deviation from theory, which assumes linear gate action. The rightmost $V_{B}$ points (gray) are obtained from the minimal $B_{\mathrm{SO}}$ in the presence of WAL. (b) Sequence at $n=6 \times 10^{11} \mathrm{~cm}^{-2}$ (upper panel) and $n=9 \times 10^{11} \mathrm{~cm}^{-2}$ (lower panel), shifted vertically for clarity. Each brown or blue marker in (a) corresponds to a trace in (b), as labeled by numerals or letters. $B_{\mathrm{SO}}$ is indicated as a guide for the eye by black dashed curves for negative $B_{Z} . B_{\mathrm{SO}}$ increases and peaks (indicating $\alpha=0$ ) before decreasing again (upper panel). Broken spin symmetry regime (lower panel): WAL is no longer suppressed here due to symmetry breaking from the cubic term at large $n$. Still, $\alpha \approx \beta$ can be identified with the narrowest WAL peak. (c) Simulation of $\alpha$ and $\beta$ along $n=6 \times 10^{11} \mathrm{~cm}^{-2}$. $\alpha$ traverses both $\beta$ (black arrow) and for smaller $\delta E_{Z}$ also zero (red arrow).

vice versa for $\alpha=-\beta$. An in-plane rotation of the PSH by a fixed angle $\pi / 2$ from $\alpha=+\beta$ to $\alpha=-\beta$ was recently demonstrated [34].

Figure 5 shows the theoretical spin diffusion length $\lambda_{\text {eff }}$ (see Appendix) and the ballistic $\lambda_{ \pm}$, together with the experimental $\lambda_{\text {SO }}$, all agreeing remarkably well. Since at $\alpha=\beta$ spin transport is ballistic despite charge diffusion, $\lambda_{-}$and its diffusive counterpart $\lambda_{\text {eff }}$ (small $\beta_{3}$ ) are essentially equivalent, as shown in SM [18]. The enhanced $\lambda_{\text {SO }}$ around $\alpha / \beta=1$ corresponds to an increased spin relaxation time $\tau_{\mathrm{SO}}=\lambda_{\mathrm{SO}}^{2} /(2 D)$. Note that $\max \left(\lambda_{+}, \lambda_{-}\right)$quantifies the deviation from the uniaxial SO field away from $\alpha=\beta$, and thus the extent to which spin rotations are not undone in a closed trajectory due to the non-Abelian nature of spin rotations around noncollinear axes. This leads to WAL, a 


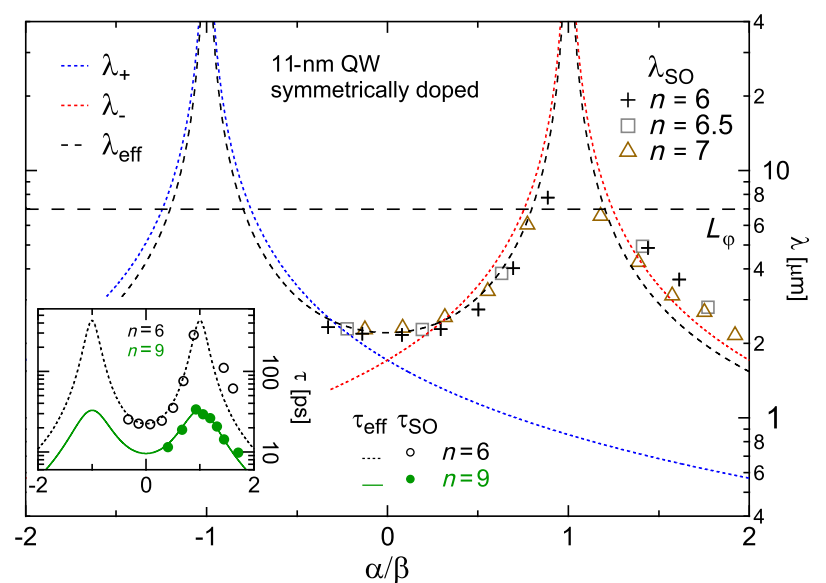

FIG. 5. Experimental and theoretical SO lengths and SO times. Experimental $\lambda_{\mathrm{SO}}=\sqrt{\hbar / 2 e B_{\mathrm{SO}}}$ (markers, densities as labeled, in units of $10^{11} \mathrm{~cm}^{-2}$ ) as a function of the dimensionless ratio $\alpha / \beta$ (from SO simulation). The ballistic $\lambda_{ \pm}$(blue and red dashes) and effective $\lambda_{\text {eff }}$ (black dashed curve) are only weakly $n$ dependent (small $\beta_{3}$ ) when plotted against $\alpha / \beta$. Thus, curves for only one density $\left(n=6 \times 10^{11} \mathrm{~cm}^{-2}\right)$ are shown. The experimental uncertainty on $\lambda_{\text {SO }}$ is captured by the spread given by the three slightly different densities. The coherence length $L_{\varphi} \approx 7 \mu \mathrm{m}$ is added for illustration (obtained from WL curves), setting the visibility of SO effects on the conductance and thus the width of the WAL-WL-WAL transition. Inset: Experimental spin relaxation time $\tau_{\mathrm{SO}}=\lambda_{\mathrm{SO}}^{2} /(2 D)$ (circles) as a function of $\alpha / \beta$ for two densities as indicated. Theory curves $\tau_{\text {eff }}$ (dashed) now include the symmetry-breaking third-harmonic term, preventing divergence at $\alpha / \beta=1$, while $\lambda_{\text {eff }}$ (main panel) does not.

finite $B_{\mathrm{SO}}$, and $\lambda_{\mathrm{SO}} \simeq \max \left(\lambda_{+}, \lambda_{-}\right)$, as observed (see Fig. 5). Unlike the corresponding time scales, the SO lengths are only weakly dependent on density and mobility when plotted against $\alpha / \beta$, allowing a comparison of various densities.

The third-harmonic contribution of cubic-in- $k$ term causes spin relaxation even at $\alpha=\beta$ and becomes visible at large densities: WAL is present in all traces and through $\alpha=\beta$ [Fig. 4(b), lower panel], because the SO field can no longer be made uniaxial, thus breaking spin symmetry and reviving WAL. A partial symmetry restoration is still apparent, where-in contrast to the $\alpha=0$ case-a minimal $B_{\mathrm{SO}}$ is reached (dashed curves) consistent with $\alpha=\beta$ [gray markers Fig. 4(a) at large $n$ ]. We include the cubic $\beta_{3}$ in the spin-relaxation time $\tau_{\text {eff }}$ (see Appendix), shown in the inset of Fig. 5 for two densities, finding good agreement with the experimental $\tau_{\mathrm{SO}}=\lambda_{\mathrm{SO}}^{2} /(2 D)$, where $D$ is the diffusion constant. Over the whole locked regime of Fig. 3(b), WAL is absent, and $\tau_{\mathrm{SO}}$ is enhanced between 1 and 2 orders of magnitude compared to $\alpha=0$. Finally, the coherence length $L_{\varphi}$ sets an upper limit for the visibility of SO effects: WAL is suppressed for $\lambda_{\text {eff }} \gg L_{\varphi}$, setting the width of the WAL-WL-WAL transition (see SM [18]).

Final remarks and outlook.-This work is laying the foundation for a new generation of experiments benefiting from unprecedented command over SO coupling in semiconductor nanostructures such as quantum wires, quantum dots, and electron spin qubits. Moreover, our work relaxes the stringency (i.e., the "fine-tuning") of the $\alpha=\beta$ symmetry condition at a particular singular point (gate) by introducing a "continuous locking" of the SO couplings $\alpha\left(V_{T}, V_{B}\right)=\beta\left(V_{T}, V_{B}\right)$ over a wide range of voltages, which should enable new experiments exciting persistent spin helices with variable pitches in GaAs wells $[12,13]$, i.e., stretchable PSHs. Further, this concept is also applicable to a range of other III-V semiconductors in various suitable configurations. Another possibility is the generation of a Skyrmion lattice (crossed spin helices) with variable lattice constants, as recently proposed in Ref. [35].

Finally, we stress that within the continuously locked regime of SO couplings we demonstrate in our study, SO-coupled quantum transport in our samples shows a very distinctive feature: it is diffusive (2D) for charge while ballistic (1D) for spins, thus providing a unique setting for coherent spin control. This ultimately adds a new functionality to the nonballistic spin transistor of Ref. [10]; i.e., it can now be made to operate as the ideal (ballistic) Datta-Das spin transistor-but in a realistic 2D diffusive system, with yet controlled spin rotations protected from spin decay.

\section{ACKNOWLEDGMENTS}

We would like to thank A. C. Gossard, D. Loss, D. L. Maslov, and G. Salis for valuable inputs and stimulating discussions. This work was supported by the Swiss Nanoscience Institute (SNI), NCCR QSIT, Swiss NSF, ERC starting grant, EU-FP7 SOLID, and MICROKELVIN, U.S. NSF DMR-1306300 and NSF MRSEC DMR1420709 and ONR N00014-15-1-2369, Brazilian grants FAPESP (SPRINT program), CNPq, PRP/USP (QNANO), and natural science foundation of China (Grant No. 11004120).

F. D., J. F., P. J. W., J.C. E., and D. M. Z. designed the experiments, analyzed the data. and co-wrote the paper. All authors discussed the results and commented on the manuscript. S. M. and D. D. A. designed, simulated, and carried out the molecular beam epitaxy growth of the heterostructures. F. D. processed the samples and with P. J. W. performed the experiments. J. F. and J. C. E. developed and carried out the simulations and theoretical work.

\section{APPENDIX: MATERIALS AND METHODS}

\section{GaAs quantum well materials}

The wells are grown on an $n$-doped substrate (for details see SM [18]) and fabricated into Hall bar structures [see inset of Fig. 2(a)] using standard photolithographic methods. The 2D gas is contacted by thermally annealed $\mathrm{GeAu} / \mathrm{Pt}$ Ohmic contacts, optimized for a low contact resistance while maintaining high back gate tunability (low leakage currents) and avoiding short circuits to the back gate. On one segment of the Hall bar, a Ti/Au top gate with 
dimensions of $300 \times 100 \mu \mathrm{m}^{2}$ is deposited. The average gate-induced $E$-field change in the well is defined as $\delta E_{Z}=1 / 2\left(V_{T} / d_{T}-V_{B} / d_{B}\right)$, with effective distance $d_{T / B}$ from the well to the top gate or back gate, respectively, extracted using a capacitor model, consistent with the full quantum description (see SM [18]). Contours of constant density follow $\delta V_{T} / d_{T}=-\delta V_{B} / d_{B}$. Deviations from linear behavior appear at most positive or negative gate voltages due to incipient gate leakage and hysteresis.

\section{Low-temperature electronic measurements}

The experiments are performed in a dilution refrigerator with base temperature $20 \mathrm{mK}$. We use a standard four-wire lock-in technique at $133 \mathrm{~Hz}$ and $100 \mathrm{nA}$ current bias, chosen to avoid self-heating while maximizing the signal. The density is determined with Hall measurements in the classical regime, whereas Shubnikov-de Haas oscillations are used to exclude occupation of the second subband, which is the case for all the data we discuss. The WAL signature is a small correction $\left(10^{-3}\right)$ to total conductance. To achieve a satisfactory signal-to-noise ratio, longitudinal conductivity traces $\Delta \sigma / \sigma_{0}=[\sigma(B)-\sigma(0)] / \sigma(0)$ are measured at least 10 times and averaged.

\section{Numerical simulations}

The simulations calculate the Rashba coefficient $\alpha$ and $\left\langle k_{z}^{2}\right\rangle$ based on the bulk semiconductor band parameters, the well structure, the measured electron densities, and the measured gate lever arms. We solve the Schrödinger and Poisson equations self-consistently ("Hartree approximation"), obtain the self-consistent eigenfunctions, and then determine $\alpha$ via appropriate expectation values [31]. The Dresselhaus coefficient $\gamma$ is extracted from fits of the simulation to the experiment, which detects the absence of WAL at $\alpha=\beta=\gamma\left(\left\langle k_{z}^{2}\right\rangle-k_{F}^{2} / 4\right)$. Thus, given $\alpha$ and $\left\langle k_{z}^{2}\right\rangle$ from the simulation and the measured $n=k_{F}^{2} /(2 \pi)$, we obtain $\gamma=11.6 \pm 1 \mathrm{eV} \AA^{3}$ consistently for all asymmetrically doped wells. Taking into account the uncertainties of the band parameters, the experimental errors, and a negligible uncertainty on $\left\langle k_{z}^{2}\right\rangle$, an overall uncertainty of about $9 \%-10 \%$ or about $\pm 1 \mathrm{eV} \AA^{3}$ on $\gamma$ results. About $1 \%-2 \%$ error originates from the experimental uncertainty of determining $\alpha=\beta$. The doping distribution (above or below well) is not expected to influence $\gamma$, and hence we use the same $\gamma$ for the more symmetrically doped wafer. Fits to the $\alpha=\beta$ experimental points then determine how much charge effectively comes from upper rather than lower doping layers, fixing the last unknown parameter also for the more symmetrically doped well (see SM [18]).

\section{Spin-dephasing times and lengths}

In WL or WAL measurements, additional spin dephasing is introduced by the external magnetic field $B$ via the AharonovBohm phase arising from the magnetic flux enclosed by the time-reversed trajectories: $\Delta \varphi=2 e A B / \hbar$, where $A$ is the loop area. Here, we take $A=\lambda_{\mathrm{SO}}^{2}=2 D \tau_{\mathrm{SO}}$ as a characteristic "diffusion area" probed by our WL or WAL experiment, with $\tau_{\mathrm{SO}}$ being the spin-dephasing time, and $\lambda_{\mathrm{SO}}$ the spin-diffusion length. By taking $\Delta \varphi=1(\mathrm{rad})$ at $B=B_{\mathrm{SO}}$, we can extract the spin-diffusion length $\lambda_{\text {SO }}$ and spin-dephasing time $\tau_{\mathrm{SO}}$ from the minima of the WAL curves from $\lambda_{\mathrm{SO}}=\sqrt{\hbar / 2 e B_{\mathrm{SO}}}$ and $\tau_{\mathrm{SO}}=\hbar\left(4 e D B_{\mathrm{SO}}\right)^{-1}$, respectively. The factor of 4 here stems from the two time-reversed paths and the diffusion length.

\section{Effective SO times and lengths}

Theoretically, we determine $\tau_{\text {SO }}$ via a spin random walk process [D'yakonov-Perel (DP)]. The initial electron spin in a loop can point (with equal probability) along the $s_{x_{-}}$, $s_{x_{+}}$, and $s_{z}$ axes (analogous to $x_{+}, x_{-}$, and $z$, respectively), which have unequal spin-dephasing times $\tau_{\mathrm{DP}, s_{x_{-}}}, \tau_{\mathrm{DP}, s_{x_{+}}}$, and $\tau_{\mathrm{DP}, s_{z}}$. For unpolarized, independent spins, we take the average $\tau_{\text {eff }}=\left(\tau_{\mathrm{DP}, s_{x_{-}}}+\tau_{\mathrm{DP}, s_{x_{+}}}+\tau_{\mathrm{DP}, s_{z}}\right) / 3$, which leads to an effective spin-difusion length $\lambda_{\text {eff }}=\sqrt{2 D \tau_{\text {eff }}}$. Actually, $\lambda_{\text {eff }}$ is defined from the average variance $\lambda_{\text {eff }}^{2}=\bar{\sigma}^{2}=2 D \tau_{\text {eff }}$, obtained by averaging the spindependent variances $\sigma_{s_{x_{-}}}^{2}=2 D \tau_{\mathrm{DP}, s_{x_{-}}}, \sigma_{s_{x_{+}}}^{2}=2 D \tau_{\mathrm{DP}, s_{x_{+}}}$, and $\sigma_{s_{z}}^{2}=2 D \tau_{\mathrm{DP}, s_{z}}$ over the spin directions $s_{x_{+}}, s_{x_{-}}$, and $s_{z}$ (this is equivalent to averaging over the $\tau$ 's and not over $1 / \tau$ 's). In the SM [18], we discuss the spin random walk and provide expressions for the DP times including corrections due to the cubic $\beta_{3}$ term. Figure 5 shows curves for the spin-dephasing times and lengths presented here. In the main panel, the cubic $\beta_{3}$ is neglected in $\lambda_{\text {eff }}$ since for $n \leq 7 \times 10^{11} \mathrm{~cm}^{-2}$, WL appears at $\alpha=\beta$ (small $\beta_{3}$ ). In contrast, the cubic term is included in $\tau_{\text {eff }}$ in the inset since at the higher density, $n=9 \times 10^{11} \mathrm{~cm}^{-2}$, WAL persists (sufficiently strong $\beta_{3}$ ).

[1] J. Sinova, S. O. Valenzuela, J. Wunderlich, C. Back, and T. Jungwirth, Spin Hall Effects, Rev. Mod. Phys. 87, 1213 (2015).

[2] V. Mourik, K. Zuo, S. M. Frolov, S. R. Plissard, E. P. A. M. Bakkers, and L. P. Kouwenhoven, Signatures of Majorana Fermions in Hybrid Superconductor-Semiconductor Nanowire Devices, Science 336, 1003 (2012).

[3] X. Wan, A. M. Turner, A. Vishwanath, and S. Y. Savrasov, Topological Semimetal and Fermi-Arc Surface States in the Electronic Structure of Pyrochlore Iridates, Phys. Rev. B 83, 205101 (2011).

[4] G. Engels, J. Lange, T. Schäpers, and H. Lüth, Experimental and Theoretical Approach to Spin Splitting in ModulationDoped $\operatorname{In}_{x} \mathrm{Ga}_{(1-x)}$ As/InP Quantum Wells for $B \rightarrow 0$, Phys. Rev. B 55, R1958 (1997).

[5] J. Nitta, T. Akazaki, H. Takayanagi, and T. Enoki, Gate Control of Spin-Orbit Interaction in an Inverted 
$\mathrm{In}_{0.53} \mathrm{Ga}_{0.47} \mathrm{As} / \mathrm{In}_{0.52} \mathrm{Al}_{0.48} \mathrm{As}$ Heterostructure, Phys. Rev. Lett. 78, 1335 (1997).

[6] P. Chuang, S.-C. Ho, L. W. Smith, F. Sfigakis, M. Pepper, C.-H. Chen, J.-C. Fan, J. P. Griffiths, I. Farrer, H. E. Beere, G. A. C. Jones, D. A. Ritchie, and T.-M. Chen, All-Electric All-Semiconductor Spin Field-Effect Transistors, Nat. Nanotechnol. 10, 35 (2015).

[7] S. Datta and B. Das, Electronic Analog of the Electro-Optic Modulator, Appl. Phys. Lett. 56, 665 (1990).

[8] Y. A. Bychkov and E. I. Rashba, Properties of a 2D Electron Gas with Lifted Spectral Degeneracy, JETP Lett. 39, 78 (1984).

[9] G. Dresselhaus, Spin-Orbit Coupling Effects in Zinc Blende Structures, Phys. Rev. 100, 580 (1955).

[10] J. Schliemann, J. C. Egues, and D. Loss, Nonballistic SpinField-Effect Transistor, Phys. Rev. Lett. 90, 146801 (2003).

[11] B. A. Bernevig, J. Orenstein, and S.-C. Zhang, Exact SU(2) Symmetry and Persistent Spin Helix in a Spin-Orbit Coupled System, Phys. Rev. Lett. 97, 236601 (2006).

[12] J. D. Koralek, C. P. Weber, J. Orenstein, B. A. Bernevig, S.-C. Zhang, S. Mack, and D. D. Awschalom, Emergence of the Persistent Spin Helix in Semiconductor Quantum Wells, Nature (London) 458, 610 (2009).

[13] M. P. Walser, C. Reichl, W. Wegscheider, and G. Salis, Direct Mapping of the Formation of a Persistent Spin Helix, Nat. Phys. 8, 757 (2012).

[14] M. Kohda, V. Lechner, Y. Kunihashi, T. Dollinger, P. Olbrich, C. Schönhuber, I. Caspers, V. V. Bel'kov, L. E. Golub, D. Weiss, K. Richter, J. Nitta, and S. D. Ganichev, Gate-Controlled Persistent Spin Helix State in (In,Ga)As Quantum Wells, Phys. Rev. B 86, 081306 (2012).

[15] S. V. Iordanskii, Y. B. Lyanda-Geller, and G. E. Pikus, Weak Localization in Quantum Wells with Spin-Orbit Interaction, JETP Lett. 60, 206 (1994).

[16] Y. Kunihashi, H. Sanada, H. Gotoh, K. Onomitsu, M. Kohda, J. Nitta, and T. Sogawa, Drift Transport of Helical Spin Coherence with Tailored Spin-Orbit Interactions, Nat. Commun. 7, 10722 (2016).

[17] H. Sanada, Y. Kunihashi, H. Gotoh, K. Onomitsu, M. Kohda, J. Nitta, P. V. Santos, and T. Sogawa, Manipulation of Mobile Spin Coherence Using Magnetic-Field-Free Electron Spin Resonance, Nat. Phys. 9, 280 (2013).

[18] See Supplemental Material at http://link.aps.org/ supplemental/10.1103/PhysRevX.7.031010 for further details on the wafer structure, temperature dependence, numerical simulations, other SO terms, effective SO fields, diffusive SO times and lengths, and Shubnikov-de Haas oscillations.

[19] S. J. Papadakis, E. P. De Poortere, H. C. Manoharan, M. Shayegan, and R. Winkler, The Effect of Spin Splitting on the Metallic Behavior of a Two-Dimensional System, Science 283, 2056 (1999).

[20] D. Grundler, Large Rashba Splitting in InAs Quantum Wells due to Electron Wave Function Penetration into the Barrier Layers, Phys. Rev. Lett. 84, 6074 (2000).
[21] M. M. Glazov and E. Y. Sherman, Nonexponential Spin Relaxation in Magnetic Fields in Quantum Wells with Random Spin-Orbit Coupling, Phys. Rev. B 71, 241312(R) (2005).

[22] E. Y. Sherman and J. Sinova, Physical Limits of the Ballistic and Nonballistic Spin-Field-Effect Transistor: Spin Dynamics in Remote-Doped Structures, Phys. Rev. B 72, 075318 (2005).

[23] F. G. Pikus and G. E. Pikus, Conduction-Band Spin Splitting and Negative Magnetoresistance in $A_{3} B_{5}$ Heterostructures, Phys. Rev. B 51, 16928 (1995).

[24] J. Fabian, A. Matos-Abiague, C. Ertler, P. Stano, and I. Žutić, Semiconductor Spintronics, Acta Phys. Slovaca 57, 565 (2007).

[25] J. Luo, H. Munekata, F. F. Fang, and P. J. Stiles, Effects of Inversion Asymmetries on Electron Energy Band Structures in GaSb/InAs/GaSb Quantum Wells, Phys. Rev. B 41, 7685 (1990).

[26] G. Bergmann, Weak Localization in Thin Films: A Time-ofFlight Experiment with Conduction Electrons, Phys. Rep. 107, 1 (1984).

[27] B. L. Altschuler and A. G. Aronov, in Electron-Electron Interactions In Disordered Conductors, edited by A. L. Efros and M. Pollak (North-Holland, Amsterdam, 1985).

[28] W. Knap, C. Skierbiszewski, A. Zduniak, E. LitwinStaszewska, D. Bertho, F. Kobbi, J. L. Robert, G. E. Pikus, F. G. Pikus, S. V. Iordanskii, V. Mosser, K. Zekentes, and Y. B. Lyanda-Geller, Weak Antilocalization and Spin Precession in Quantum Wells, Phys. Rev. B 53, 3912 (1996).

[29] J. B. Miller, D. M. Zumbühl, C. M. Marcus, Y. B. LyandaGeller, D. Goldhaber-Gordon, K. Campman, and A. C. Gossard, Gate-Controlled Spin-Orbit Quantum Interference Effects in Lateral Transport, Phys. Rev. Lett. 90, 076807 (2003).

[30] W. Wang and J. Y. Fu, Temperature Dependence of the Rashba and Dresselhaus Spin-Orbit Interactions in GaAs Wells, Physica (Amsterdam) 482B, 14 (2016).

[31] R. S. Calsaverini, E. Bernardes, J. C. Egues, and D. Loss, Intersubband-Induced Spin-Orbit Interaction in Quantum Wells, Phys. Rev. B 78, 155313 (2008).

[32] J. J. Krich and B. I. Halperin, Cubic Dresselhaus Spin-Orbit Coupling in 2D Electron Quantum Dots, Phys. Rev. Lett. 98, 226802 (2007).

[33] M. P. Walser, U. Siegenthaler, V. Lechner, D. Schuh, S. D. Ganichev, W. Wegscheider, and G. Salis, Dependence of the Dresselhaus Spin-Orbit Interaction on the Quantum Well Width, Phys. Rev. B 86, 195309 (2012).

[34] K. Yoshizumi, A. Sasaki, M. Kohda, and J. Nitta, Gate-Controlled Switching between Persistent and Inverse Persistent Spin Helix States, Appl. Phys. Lett. 108, 132402 (2016).

[35] J. Fu, P. H. Penteado, M. O. Hachiya, D. Loss, and J. C. Egues, Persistent Skyrmion Lattice of Noninteracting Electrons with Spin-Orbit Coupling, Phys. Rev. Lett. 117, 226401 (2016). 